\title{
Las relaciones entre innovación, nuevas formas de organización del trabajo y politicas de recursos humanos: el caso de la industria asturiana ${ }^{1}$
}

\author{
ISABEL GARCÍA \\ Universidad de Oviedo \\ igarcia@uniovi.es
}

Recibido: 30.06 .2008

Aceptado: 26.05.2009

\section{INTRODUCCIÓN}

En la última década estamos asistiendo al auge de los estudios sobre la innovación; un interés que no es exclusivo de las ciencias económicas o administrativas sino que ha ido desplazándose hacia el campo de la psicología y la sociología. Hoy en día, la innovación se ha convertido en una variable empresarial clave, ya que el endurecimiento de la competitividad, la globalización de la economía, el rápido cambio tecnológico y la exigencia de una mayor calidad en la producción de bienes y servicios, constituyen fenómenos que obligan a las empresas a adoptar estrategias innovadoras si quieren competir con éxito en el mercado. En este entorno caracterizado por la incertidumbre, las empresas no sólo deben adaptarse a los cambios sino que deben incluso generarlos de cara a la obtención de ventajas competitivas. Así, la creación y comercialización de nuevos productos, la mejora de los procesos productivos y la flexibilidad y capacidad de adaptación a las exigencias del mercado constituyen en la actualidad los factores más importantes de competitividad de las empresas (Villavicencio 2000).

La relevancia de la innovación no se refiere únicamente a la dimensión tecnológica. Cada vez son más frecuentes las voces que apuntan a la necesidad de que el cambio tecnológico vaya acompañado de cambios organizativos y de cambios en las políticas de recursos humanos orientados a una mayor participa-

${ }^{1}$ Este artículo se integra dentro de una investigación financiada por el Plan Nacional de $\mathrm{I}+\mathrm{D}+\mathrm{I}$ del Ministerio de Educación y Ciencia denominada «Sociedad del conocimiento y nuevos modelos de organización social del trabajo» (Proyecto SEC2002-03009).

EMPIRIA. Revista de Metodología de Ciencias Sociales. N. ${ }^{\circ}$ 17, enero-junio, 2009, pp. 63-90. ISSN: $1139-5737$ 
ción y compromiso de los trabajadores, si se quiere obtener el mayor rendimiento de las innovaciones introducidas (Baldwin y Johnson 1996; Osterman 2000). A este respecto, técnicas como la calidad total, el trabajo en equipo, la reingeniería, los grupos de mejora, figuran como prácticas que deberían incorporarse a la gestión de las empresas (Huerta Arribas 2002). De igual forma se considera necesario la existencia de una cultura y unos valores que fomenten la participación y la aportación de sugerencias e ideas por parte de los trabajadores de cara a lograr la innovación permanente (Tomás Carpi y Torrejón Velardiez 2000-2001).

Dentro de este contexto, el presente artículo se plantea precisamente si la innovación en las empresas va ligada en la práctica a la implantación de nuevas formas de organización del trabajo y a cambios en las políticas de recursos humanos, que impliquen una mayor participación y compromiso por parte de los recursos humanos. De igual forma se trata de analizar si las empresas innovadoras están potenciando valores dirigidos a fomentar una mayor participación y autonomía de los trabajadores.

La estructura del artículo es la siguiente. Después de esta introducción, en el segundo apartado se abordan las aportaciones de aquellas investigaciones que se han dedicado al análisis de las relaciones entre la capacidad innovadora de las empresas y determinadas formas de gestión de la producción y políticas de personal. Asimismo se exponen las contribuciones de los estudios que han contemplado la relación entre innovación y valores empresariales. La descripción de la situación de la innovación y de las nuevas formas de organización del trabajo en la industria asturiana se efectúa en el apartado tercero. El análisis empírico de les relaciones entre la innovación, las nuevas formas de organización del trabajo y las políticas de recursos humanos, así como las relaciones entre innovación y valores empresariales se desarrolla en los apartados cuarto y quinto. Finalmente en el sexto apartado se presentan las conclusiones más importantes del artículo.

\section{MARCO DE REFERENCIA DEL ESTUDIO}

Antes de exponer las aportaciones teóricas y empíricas que han abordado el estudio de las relaciones entre innovación y nuevas formas de gestión de la producción y de los recursos humanos, así como los valores que caracterizan a las empresas innovadoras, conviene precisar que se entiende por innovación y cuáles son sus tipos.

Una definición bastante aceptada es aquella basada en el «Manual de Frascati» de la OCDE según la cual innovación es toda aquella actividad que tiene como resultado la generación y/o comercialización de nuevos productos, nuevos procesos, así como la introducción de cambios en la gestión (organizativos, en la comercialización, en el sistema financiero, etc.) o cambios sociales que tienen que ver con el factor humano de la organización (González, Jiménez y Sáez 1997). Se trata de un concepto de innovación en sentido amplio que no queda re-

EMPIRIA. Revista de Metodología de Ciencias Sociales. N. ${ }^{\circ}$ 17, enero-junio, 2009, pp. 63-90. ISSN: $1139-5737$ 
ducido a la innovación tecnológica, la cual está relacionada únicamente con la introducción en el mercado de nuevos productos o servicios y/o la utilización de nuevos procesos de producción.

Son diversas las clasificaciones de innovación utilizadas al efecto según se haga referencia a criterios como la radicalidad de la innovación (Damanpour y Gopalakrishnan 1998) la novedad (Damanpour 1991) la naturaleza (Bhoovaraghavan Vasudevan y Rajan 1996) u otros criterios. Centrándonos en su naturaleza, la innovación puede diferenciarse en innovaciones de producto y/o servicio, innovaciones de proceso e innovaciones en métodos de gestión (Ruiz Mercader et al. 2004). Las primeras de ellas se refieren a aquellas innovaciones que introducen cambios que tienen que ver con el diseño, fabricación y comercialización de un nuevo producto y/o servicio o los que existen en el mercado pero mejorados. Por su parte, las innovaciones en procesos consisten principalmente en la introducción de nuevos procesos de producción, o la mejora de los ya existentes mediante la incorporación de nuevas tecnologías. Por último, las innovaciones de los sistemas de gestión son aquellas que tiene lugar en la estructura organizativa. Pese a esta diferenciación de los tipos de innovación según su naturaleza, hay que tener en cuanta que en la práctica tales innovaciones se relacionan entre sí y en muchas ocasiones son complementarias (Ruiz Mercader et al. 2004).

Hecha esta precisión, se puede afirmar que son limitados los estudios empíricos que se han dirigido al análisis de los cambios en la organización que tienen que ver con la implantación de nuevas formas de gestión de la producción, dentro de una estrategia innovadora de las empresas (Hailey 2001) especialmente en el ámbito español. Sin embargo, se trata de un tema ciertamente relevante ya que existe evidencia para la Unión Europea de que las empresas que están utilizando nuevas formas de organización de la producción, tales como grupos de mejora o equipos de trabajo, se las asocia con el uso intensivo de nuevas tecnologías de fabricación (Huerta Arribas 2002).

Además, la literatura que ha abordado específicamente estas relaciones señala la necesidad o conveniencia de que los cambios tecnológicos vayan acompañados de cambios en la organización del trabajo dirigidos al logro de una mayor participación de los trabajadores (Baldwin y Johnson 1996; Osterman 2000; Villavicencio 2000; Huerta Arribas 2002). Así, se considera que el cambio tecnológico y el organizativo serían estrategias que se complementan y refuerzan entre sí, y que la innovación representa no sólo la posibilidad de fabricar nuevos productos o mejorar los procesos productivos, sino también la de implantar nuevas formas de organización (Villavicencio 2000; Huerta Arribas 2002).

En general, el concepto de nuevas formas de organización del trabajo engloba una realidad bastante amplia que va desde técnicas en las que los trabajadores reflexionan sobre la producción, hasta técnicas por medio de las cuales se modifica la forma de organizar la producción. No obstante algunas de estas prácticas se consideran centrales en los sistemas avanzados de gestión (Osterman 1994). Entre ellas podemos citar los sistemas de sugerencias, los círculos de ca-

EMPIRIA. Revista de Metodología de Ciencias Sociales. N. ${ }^{\circ}$ 17, enero-junio, 2009, pp. 63-90. ISSN: 1139-5737 
lidad, los grupos de mejora, los equipos de trabajo y los sistemas de gestión de la calidad total (TQM).

Los programas que aparecieron primero en las empresas fueron los sistemas de sugerencias, seguidos por los círculos de calidad y más tarde se introdujeron los grupos de mejora (Marín García et al. 2008). Los sistemas de sugerencias individuales constituyen un procedimiento para recoger y evaluar ideas suministradas por los trabajadores de la empresa normalmente a través de un buzón de sugerencias. Por su parte, los círculos de calidad constituyen una de las prácticas más antiguas cuyo auge tuvo lugar hacia 1988 para decaer después (Castillo, Jiménez y Santos 1991). Estos círculos están constituidos por un grupo reducido de trabajadores que se reúnen voluntariamente de forma habitual para solucionar problemas que afectan sobre todo a su área de trabajo,

Los grupos de mejora comparten bastantes características con los círculos de calidad, se trata de equipos cuyos miembros se reúnen para resolver un problema concreto y cuando éste está resuelto el grupo se disuelve. Tanto los círculos de calidad como los grupos de mejora sólo tienen autonomía para proponer ideas que luego son evaluadas por un comité de mandos que son los que deciden que ideas serán implantadas. Normalmente la implantación de las ideas corre a cargo de las personas del grupo. Sin embargo los grupos de mejora se diferencian de los círculos de calidad en que la participación de sus integrantes puede no ser voluntaria, sino que son elegidos por la dirección, y tienen un carácter menos estable (Marín García et al. 2008). Además, sus miembros son elegidos por su capacitación técnica para resolver el problema y pueden pertenecer a diversos departamentos o niveles jerárquicos. Por su parte, en los equipos de trabajo los empleados asumen la responsabilidad sobre un grupo de tareas; estos equipos son responsables de un producto o de una parte del producto y tienen capacidad para tomar decisiones sobre la asignación de tareas y métodos de trabajo (Huerta Arribas 2002; Fernández, Avella y Fernández 2003).

La TQM es una técnica que consiste básicamente en que todos los miembros de la organización deben hacer su trabajo con la mayor calidad, en el menor tiempo posible y de la forma más barata (Paniagua López 1999). La TQM debe estar presente, por tanto, en todo el proceso productivo, desde que el producto se diseña y se crea hasta su lanzamiento al mercado y posterior servicio postventa; se considera que todos los departamentos y todos los trabajadores desde la alta dirección hasta los empleados están relacionados con la calidad del producto (Fernández, Avella y Fernández 2003). Sus principios básicos son: orientación al cliente (los clientes son tanto internos como externos y cada unidad organizativa es usuario y proveedor en relación a quién preceden y a quién anteceden), mejora continua, trabajo en equipo, apoyo de la alta dirección, relación estrecha con los proveedores, comunicación interna abierta tanto horizontal como vertical y desarrollo de sistemas de medición que posibiliten conocer el rendimiento de manera continua (Diéguez Castrillón 2003). Se trata de una gestión que incide en la solución de problemas y delegación de responsabilidades, de tal forma que los trabajadores deben tener la autonomía suficiente para organizar sus tareas, de ahí 
que sea frecuente la utilización de equipos de trabajo, grupos de mejora o círculos de calidad.

Si nos referimos a la relación entre los sistemas de gestión de la calidad total y las políticas de recursos humanos, este sistema de gestión pone de relieve la necesidad de un mayor grado de identificación de los trabajadores con la empresa, lo cual implica lograr un sistema de valores compartido por todos los miembros de la organización. Sin embargo, algunas investigaciones realizadas al efecto sugieren que si bien la implantación de este sistema lleva consigo una serie de modificaciones organizativas, éstas siguen manteniendo los antiguos hábitos de dirección. La participación del personal consiste en la mayor parte de los casos en reuniones y buzones de sugerencias y la autonomía «es más discursiva que real» (Brunet y Alarcon 2007:87).

Cabe mencionar como todas estas técnicas están relacionadas con un concepto que ha circulado con fuerza en la última década en ámbitos relacionados con la dirección de empresas, y es el concepto de «empowerment». Por él se entiende la delegación de poder a los empleados para que puedan actuar con más libertad para desempeñar su trabajo. Se trata de una participación centrada en la autonomía del puesto mediante la cual se persigue utilizar los conocimientos de los trabajadores en la mejora del proceso productivo, a la vez que se garantiza un mayor compromiso con los objetivos empresariales (Fernández, Avella y Fernández 2003; Lahera Sánchez 2004). Las nuevas formas de organización del trabajo antes señaladas facilitarían este proceso, dado que requieren que los trabajadores conozcan los sistemas de producción y aporten soluciones para solventar problemas (Harley 1999):

Además de las nuevas formas de organización del trabajo, también se ha considerado que la introducción de innovaciones tecnológicas u organizativas debe ir acompañada de determinados sistemas de gestión de los recursos humanos que fomenten el compromiso de los trabajadores con la empresa y su implicación en la implementación del cambio. Estos sistemas están integrados por un conjunto de políticas de recursos humanos que engloban la selección de personal, la formación, los sistemas de remuneración y la promoción interna. Atendiendo a la remuneración de los trabajadores, es de esperar que las empresas más innovadoras introduzcan estructuras retributivas que recojan no sólo un componente fijo, sino que vinculen la remuneración a los resultados de la empresa si aspiran a obtener un mayor compromiso de la mano de obra con los objetivos de la organización y con las innovaciones introducidas (Osterman 1994; Huselid y Becker 1996; Becker y Huselid 1998). Sobre este punto, se ha señalado además que un sistema que vincule los salarios al logro de los objetivos de la empresa debería acompañar a la implantación de nuevas formas de organización del trabajo (Huselid y Becker 1996; Becker y Huselid 1998).

Asimismo, existe una estrecha relación entre la innovación y la formación de los trabajadores, ya que tanto la introducción de innovaciones tecnológicas como organizativas -que conlleven una mayor iniciativa y capacidad de resolución de problemas- requieren de unos recursos humanos suficientemente cuali- 
ficados. A este respecto, diversos estudios han puesto de manifiesto que son las empresas innovadoras las que más invierten en la formación de sus recursos humanos (Osterman 1994; Baldwin y Johnson 1996; Huerta Arribas 2002; García Espejo 2008). En cuanto a la promoción interna, en las empresas innovadoras primaría el mérito (aptitudes, rendimiento, compromiso con la empresa) frente a otros criterios como la antigüedad (Osterman 1994; Becker y Huselid 1998)

Se ha indicado, por último, que la innovación requiere de un conjunto de valores orientados hacia la participación de los trabajadores, la autonomía y la capacidad de tomar decisiones y aportar ideas (Van der Panne, Van Beers y Kleinknecht 2003; Ruiz Mercader et al. 2004). Cambios tanto tecnológicos como organizativos pueden provocar resistencias por parte del personal de la empresa, ya que en principio tales cambios desestabilizan las regulaciones sociales vigentes (Villavicencio 2000). Nos referimos aquí a la existencia de culturas jerárquicas y culturas de resistencia -muy arraigadas en la empresa española- que están presentes no sólo entre los trabajadores sino también entre los mandos intermedios e incluso entre los directivos (Castillo, Jiménez y Santos 1991). Así, se indica como la introducción de innovaciones, sobre todo las que suponen una reorganización del trabajo, pueden fracasar si se introduce únicamente la técnica sin modificar los valores existentes (Ruiz Mercader et al. 2004). A este respecto se considera que los directivos de empresas innovadoras deben desarrollar estilos de dirección participativos, promover la iniciativa, la asunción de riesgos, la autonomía, la aportación de ideas y la innovación; elementos que se han caracterizado como propios de una cultura innovadora (Morcillo 1997; Tushman y O'Reilly III 2002; Urbano Pulido y Toledano Garrido 2008). Incluso el fomento de valores que potencien las innovaciones se ha llegado a calificar como crucial para la capacidad tecnológica de la empresa a largo plazo (Lester 1998).

A partir de este marco de referencia, en el presente artículo se plantea el análisis de las relaciones entre innovación, nuevas formas de organización del trabajo y políticas de recursos humanos. Asimismo, se indaga sobre la presencia o no de valores que potencien la innovación, participación y autonomía de los trabajadores en las empresas más innovadoras. Como universo de referencia se ha elegido el sector industrial, dado que es el sector más estable en sus inversiones, el que tiene mayor capacidad de absorción del progreso tecnológico y, sobre todo, porque en él radica la mayor parte de la producción de innovaciones (Molero y Buesa 1995; Barba Aragón, Aragón Sánchez y Sanz Valle 2000). Como espacio territorial del estudio se ha considerado un ámbito regional; en concreto el Principado de Asturias. Sobre este punto, diversos autores señalan la conveniencia de considerar las actividades innovadoras tomando las Comunidades $\mathrm{Au}-$ tónomas como unidades de análisis, ante la gran disparidad regional que existe en España en cuanto a innovación, y la profunda interrelación de este tipo de actividades con el entorno en el que se realizan (Costa, Duch y Lladós 2001; Buesa et al. 2002). Asimismo, la elección de Asturias resulta de interés por los fuertes procesos de reconversión, reestructuración y modernización industrial que han tenido lugar en la región en los últimos años.

EMPIRIA. Revista de Metodología de Ciencias Sociales. N. ${ }^{\circ}$ 17, enero-junio, 2009, pp. 63-90. ISSN: $1139-5737$ 


\section{LA SITUACIÓN DE LA INNOVACIÓN Y LAS NUEVAS FORMAS DE ORGANIZACIÓN DEL TRABAJO EN LA INDUSTRIA ASTURIANA}

Antes de pasar al análisis de las relaciones entre innovación, nuevas formas de organización del trabajo y políticas de recursos humanos conviene caracterizar brevemente la situación de la innovación en Asturias y el grado de implantación de las nuevas formas de organización del trabajo en el conjunto de empresas. Para ello, se ha utilizado como fuente de datos preferente la información obtenida por medio de una encuesta realizada en 2005 a empresas industriales asturianas de más de 30 trabajadores. Según el Anuario-Guía de empresas de Asturias (2004) se contabilizaron un total de 280 industrias de más de 30 trabajadores $^{2}$, obteniéndose datos de 200; cifra que representa un porcentaje muy elevado de respuesta ${ }^{3}$, el 71,4\%. Las encuestas fueron realizadas a los Directores de las empresas o en su caso a los responsables de Recursos Humanos por medio de encuestadores. La elección de estos interlocutores se justifica porque son los que en último término toman las decisiones en materia de innovación y organización de la producción, a la vez que son los que configuran las distintas políticas de recursos humanos.

Comenzando por el estado de la innovación en Asturias, hay que decir que a pesar de que se perciben signos de cambio hacia la implantación de técnicas y métodos más innovadores, la propensión empresarial a la innovación es baja (Vázquez y Lomba 2000). Así, según la Encuesta sobre Innovación Tecnológica en las Empresas (INE 2007), Asturias no figura a la cabeza de las Comunidades Autónomas según sus gastos en innovación. En concreto, su participación en innovación supuso en 2007 un 1,6\% de los gastos totales nacionales (INE 2007). Además, esta Comunidad presenta ciertas particularidades en cuanto al tipo de empresas más innovadoras. En la región asturiana se estima que el $75 \%$ de las empresas involucradas en actividades de I+D interna tienen menos de 250 trabajadores, en contraste con el total nacional, donde las PYMEs sólo realizan el 46\% del gasto (PCTI Asturias 2006-2009).

Atendiendo a los datos de la encuesta a empresas industriales, cerca de un 53\% de las industrias han desarrollado alguna innovación. En torno a un 29\% la ha patentado; una cifra relativamente baja, que sugiere que la protección de la actividad innovadora por medio del registro de patentes no es muy frecuente en España (Guarnido García y Guadamillas Gómez 1998; Gómez Uranga et al. 2008). Por último, la colaboración externa para proyectos conjuntos de innovación con otras or-

\footnotetext{
${ }^{2}$ Se consideró conveniente establecer un umbral mínimo de trabajadores y eliminar así al gran número de empresas de muy pequeño tamaño en las que es difícil que se den estrategias de innovación y políticas de recursos humanos de cierta entidad.

${ }^{3}$ En un principio, según el Anuario-Guía (versión 2004) se contabilizaron 351 empresas de las que 71 fueron rechazadas con posterioridad a razón de su activiad no industrial (62) o por tratarse de organizaciones duplicadas (9).
}

EMPIRIA. Revista de Metodología de Ciencias Sociales. N. ${ }^{\circ}$ 17, enero-junio, 2009, pp. 63-90. ISSN: $1139-5737$ 
ganizaciones como centros tecnológicos, empresas o Universidades tampoco es una práctica que esté muy extendida entre las industrias. Del total de empresas sólo el 27,4\% ha colaborado con otras entidades para actividades de I+D+I. Tal hecho apunta a que Asturias no cuenta con un sistema regional de innovación suficientemente articulado. Los agentes de este sistema son las empresas, la infraestructura de soporte a la innovación, los organismos y actuaciones públicas vinculadas a la innovación y el entorno regional y productivo de la innovación. Pues bien, se echa en falta más coordinación entre los agentes e interacción entre sus actividades, así como una mayor conexión entre la investigación y las necesidades y objetivos de las empresas; una situación que también se detecta en otras regiones españolas (Vázquez y Lomba 2000; Galende del Canto 2008).

Con relación a la implantación de nuevas formas de organización del trabajo en el conjunto de las industrias, en la encuesta realizada a empresas se incluyeron las siguientes prácticas: mejora de la calidad total (TQM), círculos de calidad, grupos de mejora continua, buzón de sugerencias y equipos de reingeniería y mejora de procesos. Las características de las tres primeras técnicas ya han sido expuestas en el apartado anterior. Respecto a los equipos de reingeniería, tales equipos estarían encargados de la revisión y rediseño de procesos y de su implementación, con el objetivo de lograr mejoras relevantes en aspectos tales como coste, calidad, servicio y tiempos de procesos (Hammer y Champy 1993). Con estas formas de organización del trabajo no se están recogiendo todas las nuevas prácticas que pueden utilizar las empresas, pero sí las más importantes y las que han sido más tratadas en la literatura.

Según los resultados obtenidos con la encuesta, las denominadas nuevas formas de organización del trabajo no están muy extendidas entre las industrias. La mejora de la calidad total está presente en el 39\% de las empresas, un 37\% ha implantado grupos de mejora continua, y los equipos de reingeniería son utilizados por el 30,4\% de las empresas. Los círculos de calidad, pese al auge que tuvieron a finales de la década de los ochenta, son ahora los menos frecuentes, un $22 \%$. La práctica más extendida es aquella que supone una menor participación de los trabajadores, el buzón de sugerencias, implantado en un 44,7\% de las empresas. Se trata de unos resultados que coinciden con los aportados por otros estudios, los cuales han mostrado cómo las nuevas formas de organización del trabajo tienen todavía una presencia limitada en nuestro país, y son los sistemas tradicionales de organización del trabajo los que siguen predominando en las empresas españolas (Huerta Arribas 2002).

\section{ANÁLISIS DE LAS RELACIONES ENTRE INNOVACIÓN, NUEVAS FORMAS DE ORGANIZACIÓN DEL TRABAJO Y POLÍTICAS DE RECURSOS HUMANOS}

El análisis empírico realizado en este apartado se basa en la encuesta a empresas industriales ubicadas en Asturias. Como paso previo al análisis es nece- 
sario seleccionar un indicador que nos mida el grado de innovación de dichas industrias.

\section{La medición de la innovación}

En la literatura dedicada a este tema se han utilizado diversos indicadores. La mayor parte de los estudios se han centrado en las empresas que desarrollan actividades de $\mathrm{I}+\mathrm{D}$. Ahora bien, la utilización de los gastos en $\mathrm{I}+\mathrm{D}$ como indicador de la innovación presenta varias y serias limitaciones. Por un lado, con este indicador se subestima la actividad innovadora de las pequeñas empresas a la vez que se obtienen resultados claramente sesgados a favor de la gran empresa. Por otro, se elimina cualquier referencia a otras actividades innovadoras que cada vez tienen mayor importancia (adquisición de bienes de equipo, realización de actividades dirigidas a la mejora de productos y procesos productivos, desarrollo de tecnologías vinculadas al procesamiento de la información). Es por ello que en la actualidad se utiliza un concepto mucho más amplio, como es el empleado por parte del INE, siguiendo las líneas marcadas por el Manual de Oslo, aunque sólo se refiere a la innovación tecnológica (Urraca 1998; Calvo 2000; INE 2007)4

Una forma alternativa de medir la innovación a los gastos de I+D es la utilización de las patentes. Sobre este indicador existe una gran controversia y ha sido objeto de frecuentes críticas. Se considera que no toda la innovación que se realiza en la empresa es patentada; las empresas pueden elegir otras formas de protección de sus invenciones que el sistema de patentes. Asimismo, la propensión a patentar puede variar según determinadas características como el tamaño, los sectores industriales, las Comunidades Autónomas e incluso los países (Coronado Guerrero y Acosta Seró 1999; Buesa et al. 2002).

Junto a estos indicadores hay que señalar otro tipo de medidas utilizadas fundamentalmente por aquellos estudios que han generado sus propios datos a partir de encuestas a empresas. En estos casos es frecuente considerar como indicador una pregunta que indaga si la empresa ha introducido innovaciones, ya sea de producto, de proceso e incluso de gestión (Costa, Duch y Lladós 2001; Huergo 2002). Se trata de analizar la innovación en un sentido amplio, sin establecer diferencias según la naturaleza de la innovación, dado que en la práctica se produce un gran solapamiento entre innovaciones de producto, de proceso y de gestión.

En esta misma línea se enmarca el presente artículo. Ahora bien, aunque en la encuesta a industrias asturianas de la que se obtienen los presentes datos

\footnotetext{
4 Así junto a los gastos en I+D se incluyen gastos en la adquisición de maquinaria y en tecnología inmaterial y de software; gastos en diseño y en ingeniería industrial; desarrollo de instalaciones para producir experimentalmente y luego normalmente; los gastos de formación y de comercialización; la reorganización de los sistemas de gestión y de producción, así como los métodos; por ejemplo, nuevos métodos de gestión de stocks y de control de calidad (INE, 2007).
} 
también se pregunta si la empresa ha realizado alguna innovación, se ha estimado más adecuado utilizar otro indicador obtenido a partir de una pregunta ordinal sobre la capacidad innovadora de la empresa; en concreto si hasta el momento la empresa ha sido más innovadora que la media del sector, con una escala de 1 a 5 , donde 1 significa «muy en desacuerdo» y 5 «muy de acuerdo». Se considera que tal medida es más acertada que la anterior, la cual está centrada en el desarrollo de una innovación que puede ser de carácter puntual, ya que indica una actividad innovadora continuada y consolidada, a la vez que permite una graduación de la respuesta.

\section{Definiciones de variables y diseño del modelo}

Una vez establecido el indicador, para analizar las relaciones entre innovación, nuevas formas de organización del trabajo y políticas de recursos humanos se ha recurrido a un modelo de regresión logística de tipo ordinal donde la variable dependiente es el indicador de innovación antes mencionado: «si se considera que la empresa ha sido hasta el momento más innovadora que la media del sector». Conviene señalar que en este artículo no se pretende la cuantificación de probabilidades. La intención de la construcción del modelo se ha centrado en el análisis de la «correlación» o «asociación» de las variables que se han considerado relevantes.

Las dimensiones y variables independientes incorporadas al modelo, relativas a las políticas de recursos humanos han sido:

- Criterios que adopta la empresa para ascender a los trabajadores. Esta dimensión está representada por cuatro variables diocotómicas referidas a diferentes criterios de promoción interna, y que son: antigüedad, rendimiento, formación reglada y formación interna.

- Criterios retributivos a partir de los cuales se determinan preferentemente los salarios. La política salarial se ha medido a través de tres variables dicotómicas: exclusivamente por lo marcado por el Convenio Colectivo, negociables individualmente y negociables colectivamente. Junto a estas variables se incluye otro indicador por medio del cual se indaga si la empresa utiliza algún tipo de retribución variable.

- Vías de reclutamiento de personal para la cobertura de los puestos. Este aspecto de la gestión de los recursos humanos se ha abordado por medio de once variables dicotómicas que hacen referencia a diversos medios de reclutamiento: empresas de trabajo temporal, empresas de selección, INEM, agencias de colocación, colegios profesionales, centros de formación, referencias personales, autocandidaturas, anuncios en prensa y a través de Internet.

- Cobertura de la formación. La intensidad de la política formativa se ha analizado por medio de una variable que mide el porcentaje de trabaja- 
dores que han sido formados en el último año (hasta el 25\%, del 26 al $50 \%$ y más del $50 \%$ ).

Para ver el grado de utilización de ciertas prácticas que pueden denominarse nuevas formas de organización del trabajo, y que en la actualidad están muy relacionadas con la calidad, se ha acudido a cinco variables dicotómicas cada una de las cuales hace referencia a una práctica concreta: mejora de la calidad total (TQM), círculos de calidad, grupos de mejora continua, buzón de sugerencias y equipos de reingeniería y mejora de procesos. Se trata de prácticas que van desde la simple recogida de opiniones de los trabajadores, hasta otras que implican una intervención directa de éstos en la organización de la producción.

Dado que la introducción de nuevas formas de organización del trabajo presupone en teoría una mayor autonomía y capacidad de decisión de los trabajadores, se ha incluido en el modelo una variable que mide el grado de descentralización de la toma de decisiones, por medio de una escala que oscila de 1 a 5 , donde 1 significa «centralización» y 5 «descentralización». Asimismo, es de suponer que estas nuevas prácticas conlleven una mayor polivalencia de los trabajadores. Para comprobar este supuesto se ha incorporado al modelo otra variable relativa al grado de polivalencia que caracteriza la fuerza laboral de la empresa, con una escala de 1 a 5, donde la polivalencia tiene el valor 1 y la especialización el valor 5 .

Sobre la estructura organizativa se han incluido dos variables: si el trabajo se desarrolla por equipos (sí, habitualmente; sí, aunque no de forma habitual; no) y el número de niveles jerárquicos. Sobre este último aspecto, es de suponer que las empresas innovadoras son entidades menos jerárquicas, que tratarán de reducir el número de niveles para aumentar así la autonomía de los trabajadores y la flexibilidad de la organización.

También se ha querido recabar información sobre los recursos estratégicos que caracterizan a las empresas más innovadoras y sobre su entorno. Los recursos estratégicos se han contemplado a partir de ocho variables ordinales con una escala de 1 a 5 , en la que 1 es «nada importante» y 5 «muy importante». Tales recursos son: precio de los productos y/o servicios, atención al cliente, calidad del producto y/o servicio, tecnología, productividad de los trabajadores, gasto en investigación y desarrollo y capacidad de innovación. Junto a estas variables se ha incorporado al modelo otra variable que mide el grado de competitividad de la empresa, con una escala de 1 a 5 , donde 1 es «muy inferior a la media del sector» y 5 «muy superior a la media del sector». El tipo de entorno se contempla a través de una variable dicotómica por medio de la cual se pregunta si la empresa se mueve en un entorno de cambios rápidos. Es de prever que cuanto mayor sea la incertidumbre y más rápidos los cambios del entorno, mayor será la necesidad de innovar.

Un aspecto que tiene que ver con las relaciones industriales y que se ha considerado relevante analizar es si en las empresas innovadoras hay órganos de representación de los trabajadores. Para ello se ha introducido en el modelo una va-

EMPIRIA. Revista de Metodología de Ciencias Sociales. N. ${ }^{\circ}$ 17, enero-junio, 2009, pp. 63-90. ISSN: $1139-5737$ 
riable dicotómica que mide sí están presentes o no tales órganos de representación. Sobre este punto se ha señalado que la actitud de los sindicatos resulta de gran importancia para favorecer o dificultar las innovaciones (Osterman 1994; Huerta Arribas 2002). Así, los sindicatos suelen desconfiar sobre todo de las innovaciones que impliquen una nueva organización del trabajo. Los motivos son varios, y van desde la pérdida de protagonismo de los interlocutores sindicales como representantes de los trabajadores, hasta la ausencia de compensación económica para los empleados ante los cambios introducidos en los puestos de trabajo.

Por último, se han incorporado al modelo tres variables de control: el tamaño de la empresa (de 30 a 50 trabajadores, de 51 a 100, más de 100), la antigüedad medida a partir del año de fundación de la organización (hasta 1970, de 1971 a 1980, de 1981 a 1990, de 1991 a 2003) y si la empresa exporta o no. En la tabla que figura a continuación sólo se presentan las variables que han resultado significativas ${ }^{5}$ (Tabla 1 ).

Tabla 1. Modelo de regresión logística ordinal

\begin{tabular}{|c|c|}
\hline № DE NIVELES JERÁRQUICOS EN LA EMPRESA & $-0,673 * * *$ \\
\hline \multicolumn{2}{|l|}{ DESARROLLO DEL TRABAJO POR EQUIPOS (Cat. Residual: No) } \\
\hline 1. Sí, habitualmente & 0,063 \\
\hline 2. Sí, aunque no de forma habitual & $2,974 * *$ \\
\hline \multicolumn{2}{|l|}{ TIPO DE ENTORNO } \\
\hline Entorno de cambios rápidos (Cat. Residual: No) & $1,184^{*}$ \\
\hline \multicolumn{2}{|l|}{ RECURSOS ESTRATÉGICOS } \\
\hline Precio de los productos y/o servicios (Escala 1 a 5 ) & $0,656^{*}$ \\
\hline Atención al cliente (Escala 1 a 5) & $-1,777 * * *$ \\
\hline Cualificación de los trabajadores (Escala 1 a 5) & $0,993 * *$ \\
\hline Capacidad de innovación (Escala 1 a 5) & $1,465 * * *$ \\
\hline GRADO DE COMPETITIVIDAD DE LA EMPRESA (Escala 1 a 5) & $2,333 * * *$ \\
\hline \multicolumn{2}{|l|}{ FORMAS DE RECLUTAMIENTO DE PERSONAL } \\
\hline Empresas de selección (Cat. Residual: No) & $1,819 * *$ \\
\hline INEM (Cat. Residual: No) & $2,227 * * *$ \\
\hline Asociaciones empresariales (Cat. Residual: No) & $-1,528 *$ \\
\hline Anuncios en prensa (Cat. Residual: No) & $-1,240 *$ \\
\hline \multicolumn{2}{|l|}{ CRITERIOS PARA ASCENSOS } \\
\hline Formación interna (Cat. Residual: No) & $1,189^{*}$ \\
\hline \multicolumn{2}{|l|}{ CRITERIOS PARA DETERMINACIÓN DE LOS SALARIOS } \\
\hline Negociables individualmente (Cat. Residual: No) & $1,834 * *$ \\
\hline \multicolumn{2}{|l|}{ NUEVAS FORMAS DE ORGANIZACIÓN DEL TRABAJO } \\
\hline Grupos de mejora continua (Cat. Residual: No) & $0,137 *$ \\
\hline Buzón de sugerencias (Cat. Residual: No) & $1,765 * *$ \\
\hline
\end{tabular}

${ }^{5}$ Se han descartado problemas de multicolinealidad entre las variables.

EMPIRIA. Revista de Metodología de Ciencias Sociales. N. ${ }^{\text {17 }}$, enero-junio, 2009, pp. 63-90. ISSN: $1139-5737$ 
PORCENTAJE DE TRABAJADORES FORMADOS

(Cat. Residual: Hasta el 25\%)

\begin{tabular}{ll}
\hline 1. Más del 50\% & $3,268^{* * *}$ \\
2. Del 26 al 50\% & $1,790^{* *}$ \\
\hline $\begin{array}{l}\text { ORGANOS DE REPRESENTACIÓN DE LOS TRABAJADORES } \\
\text { (Cat. Residual: No) }\end{array}$ & $-1,321^{*}$ \\
\hline AÑO DE FUNDACIÓN (Cat. Residual: 1991 a 2003) & $-0,751$ \\
\hline 1. Hasta 1970 & $-1,805^{*}$ \\
2. De 1971 a 1980 & 1,315 \\
\hline T. De 1981 a 1990 & \\
\hline TAMAÑO DE LA EMPRESA (Cat. Residual: Más de 100) & $3,126^{* * *}$ \\
\hline 1. De 30 a 50 trabajadores & $1,126^{*}$ \\
\hline 2. De 51 a 100 trabajadores & 200 \\
\hline
\end{tabular}

Niveles de significación: $* p<0,1000 * * p<0,0500 * * * p<0,0100$

Fuente: Encuesta a empresas industriales 2005.

\section{Discusión de resultados}

Según los resultados obtenidos, se destacan dos prácticas cuya presencia se asocia con altos niveles de innovación. La principal de ellas es una práctica meramente consultiva, el buzón de sugerencias, por medio de la cual se busca que la mano de obra aporte sus ideas para la mejora de la calidad, la reducción de costes, etc. La segunda son los grupos de mejora continua; grupos, que como ya habíamos visto, no constituyen estructuras permanentes sino que se crean para resolver problemas concretos y luego se disuelven. Tal práctica está asociada a las industrias más innovadoras, pero su nivel de significación no es muy elevado. El resto de prácticas, poco extendidas en el conjunto de empresas, no presentan una implantación distinta según el grado de innovación, y como tal no resultan variables que logren significación estadística. Parece, por tanto, que las empresas están introduciendo innovaciones tecnológicas sin que estén cambiando la organización del trabajo hacia formas más participativas.

En las empresas innovadoras la toma de decisiones no está más descentralizada que en el resto, ya que tal variable no alcanza suficiente significación estadística; es decir, no existen diferencias relevantes entre las industrias que son más innovadoras y las que no lo son. Sobre este aspecto hay que tener en cuenta que la media del conjunto de empresas para esta variable, en la escala de 1 a 5 donde 1 representa la centralización y 5 la descentralización, es de 2,2, cercana a la centralización máxima. Tal resultado indica que la división vertical del trabajo es predominante entre las empresas, e incluso que la implantación de nuevas formas de organización del trabajo, como los grupos de mejora, puede no ir acompañada de una mayor autonomía en la toma de decisiones por parte de los 
trabajadores, como ya se ha indicado en otros estudios (Harley 1999; Brunet y Alarcón 2007). Sin embargo, cuanto mayor es la innovación menor es el número de niveles jerárquicos. Este resultado muestra que las empresas innovadoras sí poseen estructuras más flexibles que facilitan la respuesta y adaptación a los cambios del entorno, aunque tal característica no lleve consigo una descentralización de las decisiones.

Respecto a otras variables relacionadas con la organización del trabajo, el desarrollo de trabajo por equipos, aunque no de forma habitual, es más probable que se desarrolle en las industrias innovadoras, un resultado que está en línea con la utilización de grupos de mejora que no constituyen una práctica permanente. No obstante, la polivalencia de la mano de obra, que teóricamente debería ser superior en estas empresas, no presenta diferencias relevantes según el grado de innovación y por ello no figura en el modelo.

$\mathrm{Si}$ nos referimos ahora a las distintas políticas de recursos humanos, comenzando por la política salarial, la negociación individual es el criterio fundamental para la determinación de los salarios conforme las empresas son más innovadoras, por encima de los convenios colectivos o la negociación colectiva. Este resultado viene a ser un indicador de la progresiva pérdida de relevancia de los mecanismos formales de negociación colectiva en nuestro país, y del creciente auge de los sistemas de remuneración individual relacionados con el rendimiento (Martínez Lucio y Simpson 1993). Respecto a si la innovación se asocia a la implantación de algún tipo de retribución variable, ligada al cumplimiento de los objetivos de la empresa, el indicador que mide esta opción no alcanza suficiente significación estadística. Por tanto, la innovación no aparece relacionada con sistemas de incentivos que potencien el compromiso de los trabajadores con los resultados de la organización o con el trabajo en equipo; un resultado puesto de manifiesto por otras investigaciones (Tomás Carpi y Torrejón Velardiez 2000-2001).

Donde se observan las diferencias más notables entre las industrias según su grado de innovación es en la política formativa. La cobertura de la formación, medida a través del porcentaje de trabajadores formados, es sensiblemente superior conforme aumenta el grado de innovación de las empresas. Y es que la innovación lleva implícita una necesaria puesta al día de los conocimientos de los trabajadores y necesita de un elevado nivel de cualificación de los recursos humanos. La relevancia de la formación se observa también en los criterios que rigen las promociones dentro de la organización. Así, el criterio fundamental por el que se regulan los ascensos es la formación interna. Respecto a las vías o canales de reclutamiento de personal, los canales que más se utilizan conforme la industria es más innovadora son las empresas de selección y sobre todo el INEM.

En línea con los resultados anteriores, las empresas innovadoras tienen en la cualificación de los trabajadores uno de los recursos estratégicos más importantes, junto con la capacidad de innovación. Esta capacidad de innovación es necesaria si tenemos en cuenta que tales organizaciones se mueven en un entorno dominado por los cambios rápidos; ante la incertidumbre que provoca un entor-

EMPIRIA. Revista de Metodología de Ciencias Sociales. N. ${ }^{\circ}$ 17, enero-junio, 2009, pp. 63-90. ISSN: 1139-5737 
no de tales características se hacen necesarios altos niveles de innovación y una elevada capacidad adaptativa. Que la empresa sea más competitiva que la media del sector, es un rasgo que aparece asociado a la innovación.

La presencia sindical figura con un coeficiente de signo negativo; es decir, las industrias más innovadoras no suelen contar con órganos de representación de los trabajadores; un hecho que favorece la discrecionalidad del empresario a la hora de organizar el trabajo y tomar decisiones. Sobre este aspecto, la dirección puede opinar que de esta forma se evita la posible oposición de los sindicatos a la introducción de innovaciones tecnológicas que conlleven un cambio en las reglas del trabajo.

$\mathrm{Si}$ atendemos por último a las variables de control, resultan significativas el tamaño y la antigüedad de la organización. Son las industrias de 51 a 100 trabajadores y sobre todo las de 30 a 50 las que más probabilidades tienen de innovar con relación a las empresas de mayor tamaño, las cuales parecen menos flexibles y menos ágiles para adaptarse a los cambios. No obstante, sobre la relación entre tamaño de la empresa e innovación las investigaciones que han abordado este aspecto no establecen resultados concluyentes (Gumbau Albert 1997). Mientras algunas señalan la mayor capacidad de la gran empresa (Yagüe 1992) otras investigaciones muestran un esfuerzo inversor superior en términos relativos entre las empresas de menor tamaño (Fariñas y Huergo 1999). Respecto a la antigüedad de la organización, las industrias fundadas antes de 1980 tienen una menor probabilidad de ser innovadoras que las creadas a partir de dicha fecha.

\section{ANÁLISIS DE LAS RELACIONES ENTRE INNOVACIÓN Y VALORES EMPRESARIALES}

Se ha relacionado el éxito en la introducción de innovaciones con el fomento por parte de la dirección de valores orientados a la participación y autonomía de los trabajadores. Así, se considera que la innovación requiere de un ambiente o cultura organizativa que la promueva y favorezca. Una cultura organizativa sustentada en valores como flexibilidad, asunción de riesgos, inquietud, creatividad, etc., hace que el proceso de innovación tenga lugar de forma más ágil y dinámica (Velasco Balmaseda y Zamanillo Elguezabal 2008).

Para analizar precisamente si las empresas innovadoras potencian este tipo de valores, se incluyeron en la encuesta una serie de enunciados que describen quince valores que pueden ser perseguidos por la organización y que están basados en el trabajo desarrollado por Quinn y colaboradores (Quinn y Rohrbauch 1983), si bien se ha incidido más en aquellos que han sido considerados por la literatura como propios de una cultura innovadora (Morcillo 1997; Tushman y O'Reilly III 2002; Ruiz Mercader et al. 2004;).

Los valores propuestos han sido: eficiencia, productividad, rentabilidad, trabajo en equipo, cohesión, relaciones humanas, crecimiento y expansión, excelencia y calidad de los resultados, innovación y cambio, control, flexibilidad 
para adaptarse, autonomía de los trabajadores, participación y discusión abierta, evaluar las preocupaciones y las ideas de los empleados y procesos creativos para resolver problemas. Para cada uno de ellos se debía señalar su importancia en una escala de 1 a 5 , donde 1 es «nada» y 5 «mucha». Por valores podemos entender «las preferencias integradas y tácitas acerca de los objetivos que la organización debe lograr y la forma cómo debería hacerlo» (De Long y Fahey 2000:115).

Para analizar la relación entre la actividad innovadora y los valores empresariales mencionados se ha utilizado como indicador de innovación la misma medida que en el análisis de regresión precedente: si se considera que la empresa ha sido hasta el momento más innovadora que la media del sector en una escala de 1 a 5 , aunque ahora se han agrupado las respuestas en tres niveles. Las puntuaciones 1 y 2 representan a las empresas con un nivel de innovación bajo, el 3 indica un nivel medio y los niveles 4 y 5 corresponden a las industrias con un nivel de innovación alto.

Una primera aproximación a los resultados se obtiene con las medias que presentan cada uno de estos valores según el nivel de innovación (Anexo Tabla 7). Para todos los grupos de empresas, los valores predominantes son los orientados a los logros de productividad, rentabilidad y eficiencia. También son importantes la consecución de la excelencia y la calidad de los resultados. Para las industrias de alta y moderada innovación sobresale asimismo la flexibilidad para adaptarse. No obstante, aparte de estas similitudes también se observan diferencias interesantes entre los grupos que van a ser analizadas mediante la realización de la prueba ANOVA de un factor. Por medio de esta técnica se ha tratado de determinar si existen diferencias estadísticamente significativas entre las empresas según su grado de innovación.

En la Tabla 2 figuran los resultados obtenidos para aquellos ítems que presentan diferencias estadísticamente significativas entre los tres grupos de empresas considerados. Como puede observarse, los valores que tienen que ver con la participación de los trabajadores (participación y discusión abierta, evaluar ideas de los trabajadores, procesos creativos para resolver problemas) y con la autonomía de los mismos presentan diferencias significativas, si bien el valor de autonomía registra una significación estadística menor que los anteriores. También hay diferencias notables en los valores de innovación, crecimiento y expansión, flexibilidad para adaptarse y excelencia y calidad de los resultados. Con una significación menor figuran los valores de cohesión y eficiencia.

Pero lo más interesante es determinar qué grupo o grupos son los que persiguen valores distintos respecto a los otros grupos, para lo cual se ha realizado un análisis Post Hoc ${ }^{6}$. En la Tabla 8 (Anexo) figuran todas las posibles combinaciones dos a dos entre los valores de la variable «nivel de innovación». Los gru-

\footnotetext{
${ }^{6}$ Se ha realizado la prueba de homogeneidad de varianzas por medio del estadístico de Levene, el cual permite contrastar la hipótesis de que las varianzas poblacionales son iguales. Asumiendo varianzas iguales, se ha aplicado el procedimiento Post hoc de Tukey.
}

EMPIRIA. Revista de Metodología de Ciencias Sociales. N. ${ }^{\circ}$ 17, enero-junio, 2009, pp. 63-90. ISSN: $1139-5737$ 
pos cuyas medias difieren significativamente al nivel de 0,05 están señalados con un asterisco. A excepción del valor relativo a la flexibilidad para adaptarse, las empresas con un nivel de innovación alto difieren significativamente de aquellas que tienen un nivel de innovación bajo; es decir, las primeras otorgan a los valores anteriormente mencionados una valoración superior. Por su parte, las industrias moderadamente innovadoras valoran más el crecimiento, la excelencia y calidad de los resultados, la innovación y el cambio, la flexibilidad para adaptarse y la participación de los trabajadores, que las empresas de un nivel bajo.

Tabla 2. Resultados del ANOVA de un factor

\begin{tabular}{|c|c|c|c|c|c|c|}
\hline & & $\begin{array}{l}\text { Suma de } \\
\text { cuadrados }\end{array}$ & $\mathrm{gl}$ & $\begin{array}{l}\text { Media } \\
\text { cuadrática }\end{array}$ & $\mathrm{F}$ & Sig. \\
\hline Eficiencia & $\begin{array}{r}\text { Inter.-grupos } \\
\text { Intra-grupos } \\
\text { Total }\end{array}$ & $\begin{array}{r}3,130 \\
78,782 \\
81,912\end{array}$ & $\begin{array}{r}2 \\
179 \\
181\end{array}$ & $\begin{array}{l}1,565 \\
0,440\end{array}$ & 3,556 & 0,031 \\
\hline Cohesión & $\begin{array}{r}\text { Inter.-grupos } \\
\text { Intra-grupos } \\
\text { Total }\end{array}$ & $\begin{array}{r}6,870 \\
187,701 \\
194,571\end{array}$ & $\begin{array}{r}2 \\
179 \\
181\end{array}$ & $\begin{array}{l}3,435 \\
1,049\end{array}$ & 3,276 & 0,040 \\
\hline Crecimiento, expansión & $\begin{array}{r}\text { Inter.-grupos } \\
\text { Intra-grupos } \\
\text { Total }\end{array}$ & $\begin{array}{r}19,374 \\
11,354 \\
130,728 \\
\end{array}$ & $\begin{array}{r}2 \\
177 \\
179\end{array}$ & $\begin{array}{l}9,687 \\
0,629\end{array}$ & 15,398 & 0,000 \\
\hline $\begin{array}{l}\text { Excelencia, calidad } \\
\text { resultados }\end{array}$ & $\begin{array}{r}\text { Inter.-grupos } \\
\text { Intra-grupos } \\
\text { Total }\end{array}$ & $\begin{array}{r}5,439 \\
898,425 \\
94,863\end{array}$ & $\begin{array}{r}2 \\
180 \\
182\end{array}$ & $\begin{array}{l}2,719 \\
0,497\end{array}$ & 5,474 & 0,005 \\
\hline Innovación y cambio & $\begin{array}{r}\text { Inter.-grupos } \\
\text { Intra-grupos } \\
\text { Total }\end{array}$ & $\begin{array}{r}17,836 \\
152,587 \\
170,423\end{array}$ & $\begin{array}{r}2 \\
179 \\
181\end{array}$ & $\begin{array}{l}8,918 \\
0,852\end{array}$ & 10,462 & $\overline{0,000}$ \\
\hline $\begin{array}{l}\text { Flexibilidad para } \\
\text { adaptarse }\end{array}$ & $\begin{array}{r}\text { Inter.-grupos } \\
\text { Intra-grupos } \\
\text { Total }\end{array}$ & $\begin{array}{r}6,786 \\
114,231 \\
121,061 \\
\end{array}$ & $\begin{array}{r}2 \\
180 \\
182\end{array}$ & $\begin{array}{l}3,393 \\
0,635\end{array}$ & 5,346 & 0,006 \\
\hline $\begin{array}{l}\text { Autonomía de los } \\
\text { trabajadores }\end{array}$ & $\begin{array}{r}\text { Inter.-grupos } \\
\text { Intra-grupos } \\
\text { Total }\end{array}$ & $\begin{array}{r}4.599 \\
154.395 \\
159.650\end{array}$ & $\begin{array}{r}2 \\
180 \\
182\end{array}$ & $\begin{array}{l}2.300 \\
0,858\end{array}$ & 2.681 & 0,071 \\
\hline Participación, discusión & $\begin{array}{r}\text { Inter.-grupos } \\
\text { Intra-grupos } \\
\text { Total }\end{array}$ & $\begin{array}{r}9,150 \\
150,500 \\
159,650\end{array}$ & $\begin{array}{r}2 \\
180 \\
182\end{array}$ & $\begin{array}{l}4,575 \\
0,836\end{array}$ & 5,472 & 0,005 \\
\hline $\begin{array}{l}\text { Evaluar ideas de los } \\
\text { trabajadores }\end{array}$ & $\begin{array}{r}\text { Inter.-grupos } \\
\text { Intra-grupos } \\
\text { Total }\end{array}$ & $\begin{array}{r}5,464 \\
149,563 \\
155,027 \\
\end{array}$ & $\begin{array}{r}2 \\
180 \\
182\end{array}$ & $\begin{array}{l}2,732 \\
0,831\end{array}$ & 3,288 & 0,040 \\
\hline $\begin{array}{l}\text { Procesos creativo paras } \\
\text { resolver problemas }\end{array}$ & $\begin{array}{r}\text { Inter.-grupos } \\
\text { Intra-grupos } \\
\text { Total }\end{array}$ & $\begin{array}{r}6,226 \\
163,274 \\
169,500\end{array}$ & $\begin{array}{r}2 \\
179 \\
181\end{array}$ & $\begin{array}{l}3,113 \\
0,912\end{array}$ & 3,413 & 0,035 \\
\hline
\end{tabular}

Fuente: Encuesta a empresas industriales 2005. 
Los resultados de los análisis efectuados parecen verificar, por tanto, la idea de que en las empresas innovadoras se fomentan aquellos valores que promueven la participación de los trabajadores en mayor medida que en el resto. Esta afirmación no parece tan extensible a la autonomía de la mano de obra, ya que las diferencias entre grupos referidas a esta dimensión no alcanzan una significación muy elevada.

Un análisis complementario a la prueba ANOVA de un factor consiste en tratar las puntuaciones de todos los valores de forma simultánea por medio de un análisis factorial de componentes principales. Esta técnica de reducción de datos sirve para encontrar grupos homogéneos de variables a partir de un conjunto más numeroso. Estos grupos homogéneos se forman con las variables que correlacionan mucho entre sí; de esta manera, el conjunto de quince valores puede reducirse a un menor número de dimensiones que resumen la importancia de los valores propuestos.

Atendiendo a los indicadores de bondad del análisis factorial (Tabla 3) se comprueba que tales valores no son independientes sino que están relacionados entre sí. Así, el índice de Kayser Meyer Olkin es próximo a uno y el test de esferidad de Barlett permite rechazar la hipótesis de que la matriz de correlaciones de todas las variables es una matriz de identidad.

Tabla 3. Índices de bondad del análisis factorial

\begin{tabular}{|l|l|}
\hline Índice de Kayser Meyer Olkin & 0,880 \\
\hline Test de esfericidad de Barlett & \\
- Chi-cuadrado & 1125,205 \\
- Nivel de significación & 0,000 \\
\hline
\end{tabular}

Fuente: Encuesta a empresas industriales 2005.

Producto del análisis factorial se deriva que los quince valores propuestos inicialmente se pueden agrupar y resumir en tres dimensiones que explican el $58,3 \%$ de la varianza de los datos originales (Tabla 4). Como criterio para decidir el número de factores se ha aplicado la Regla de Kaiser, por la que se extraen aquellos factores cuyos autovalores son mayores que la unidad.

Tabla 4. Resultados del análisis factorial

\begin{tabular}{|l|c|c|}
\hline Dimensión & \% de la varianza & \% acumulado \\
\hline 1 & 38,94 & 38,94 \\
\hline 2 & 12,49 & 51,43 \\
\hline 3 & 6,89 & 58,33 \\
\hline
\end{tabular}

Fuente: Encuesta a empresas industriales 2005. 
Para facilitar la interpretación de estas dimensiones se ha acudido a la matriz de componentes rotados $^{7}$ (Tabla 5). Las puntuaciones factoriales incluidas en la matriz explican la relación entre cada valor y las nuevas dimensiones. Cada bloque contiene aquel conjunto de variables que presentan máxima saturación en valor absoluto sobre un mismo factor. Dentro de cada bloque las variables se disponen de mayor a menor saturación.

Tabla 5. Matriz de componentes rotados

\begin{tabular}{lccc}
\hline IMPORTANCIA & 1 & 2 & 3 \\
\hline Procesos creativos para resolver problemas & 0,727 & 0,372 & $-0,001$ \\
Participación, discusión abierta & 0,713 & 0,417 & $-0,069$ \\
Innovación y cambio & 0,711 & 0,254 & 0,208 \\
Autonomía de los trabajadores & 0,696 & 0,351 & $-0,014$ \\
Evaluar ideas de los trabajadores & 0,654 & 0,497 & $-0,095$ \\
Crecimiento y expansión & 0,644 & $-0,090$ & 0,406 \\
Excelencia y calidad de los resultados & 0,571 & 0,127 & 0,462 \\
Flexibilidad ‘para adaptarse & 0,417 & 0,398 & 0,253 \\
Relaciones Humanas & 0,252 & 0,790 & 0,128 \\
Trabajo en equipo & 0,303 & 0,728 & 0,199 \\
Cohesión & 0,308 & 0,645 & 0,059 \\
Productividad & 0,048 & 0,062 & 0,809 \\
Rentabilidad & 0,039 & 0,027 & 0,752 \\
Eficiencia & $-0,069$ & 0,443 & 0,566 \\
Control & 0,340 & 0,136 & 0,433 \\
\hline
\end{tabular}

Fuente: Encuesta a empresas industriales 2005.

La primera dimensión está vinculada con las variables: procesos creativos para resolver problemas; participación y discusión abierta; innovación y cambio; autonomía de los trabajadores; evaluar ideas de los trabajadores; crecimiento y expansión; excelencia y calidad de los resultados y flexiblidad para adaptarse. Se trata de valores relacionados sobre todo con la innovación y la implicación de los trabajadores. El segundo factor o dimensión está vinculado con las relaciones humanas, el trabajo en equipo y la cohesión; por ello se le ha denominado relaciones humanas y trabajo en equipo. Por último, el tercer factor recoge los valores de productividad, rentabilidad, eficiencia y control; valores relacionados con lo que se ha llamado resultados empresariales.

Una vez lograda la solución factorial, se han obtenido las puntuaciones para cada sujeto en cada uno de los factores obtenidos. Para la estimación de estas puntuaciones factoriales se ha aplicado el método de Regresión. Posteriormente

\footnotetext{
${ }^{7}$ El método de rotación utilizado es el método Varimax que es un tipo de rotación ortogonal de los factores que trata de minimizar el número de variables que tienen saturaciones altas en cada factor. El objetivo de la rotación de los factores es obtener una solución más interpretable, en el sentido de que las variables muy correlacionadas entre sí presenten saturaciones altas sobre un mismo factor y bajas sobre el resto.
} 
se han correlacionado las puntuaciones de cada factor con la variable que mide el nivel de innovación mediante el coeficiente de correlación de Pearson (Tabla 6). Como puede observarse, existe una asociación positiva y significativa entre el nivel de innovación y el factor denominado innovación e implicación de los trabajadores. Igualmente positiva es la correlación entre el nivel de innovación y el factor que agrupa a los valores relacionados con los resultados empresariales, si bien el valor del coeficiente es muy bajo y de menor significación. Por último, no existe ningún tipo de asociación con el factor que aglutina los valores de relaciones humanas y trabajo en equipo.

Tabla 6. Innovación y valores empresariales

\begin{tabular}{|l|l|}
\hline & $\begin{array}{l}\text { Nivel de innovación } \\
\text { Correlación de Pearson }\end{array}$ \\
\hline FACTOR 1 & $0,373 * * *$ \\
\hline FACTOR 2 & 0,063 \\
\hline FACTOR 3 & $0.162 * *$ \\
\hline
\end{tabular}

Niveles de significación: $* p<0,1000 * * p<0,0500 * * * p<0,0100$

Fuente: Encuesta a empresas industriales 2005.

\section{CONCLUSIONES}

A lo largo de este artículo se ha analizado las relaciones entre la capacidad innovadora de las empresas y la implementación de nuevas formas de organización del trabajo y de ciertas políticas de recursos humanos orientadas a una mayor participación, autonomía y compromiso de los trabajadores. Según los resultados obtenidos, la probabilidad de acudir a nuevas formas de organización de la producción es mayor conforme más elevado es el nivel de innovación de las empresas. No obstante, las prácticas que figuran más asociadas a este tipo de organizaciones son, sobre todo, las que tienen un carácter meramente consultivo, orientadas a que los trabajadores reflexionen sobre la producción y aporten sus ideas. Los grupos de mejora, que presuponen el paso de un trabajo individual a un trabajo de tipo grupal, aunque sea de forma temporal, también figuran asociados a la innovación pero en menor medida. Como señala Castillo (2000), frente al imaginario difundido de que la innovación tecnológica lleva consigo automáticamente nuevas formas de organización del trabajo, la realidad muestra que esta afirmación responde muchas veces más a un «deseo» que a una constatación empírica.

En las empresas innovadoras la toma de decisiones no está más descentralizada que en el resto de empresas. Aunque poseen una estructura más flexible, con un menor número de niveles jerárquicos que facilita la adaptación a los cambios de un entorno caracterizado precisamente por los cambios rápidos, e inclu-

EMPIRIA. Revista de Metodología de Ciencias Sociales. N. ${ }^{\text {17 }}$, enero-junio, 2009, pp. 63-90. ISSN: $1139-5737$ 
so aunque introduzcan ciertas prácticas que busquen una mayor implicación de los trabajadores con los objetivos de la empresa, la toma de decisiones sigue estando muy centralizada; en manos de la gerencia y los directivos. La división vertical del trabajo permanece, por tanto, en estas organizaciones.

Si nos referimos a ciertas políticas de recursos humanos que pueden potenciar asimismo el compromiso de los trabajadores y su implicación en la implementación del cambio, en las empresas innovadoras tienen poca importancia los sistemas de incentivos que presuponen la variabilidad de la masa salarial y la asocian al logro de los objetivos de la empresa y del trabajo en equipo. Sin embargo, se trata de sistemas que la literatura ha señalado de gran importancia de cara a la implantación de nuevas prácticas de gestión de la producción. Sí sobresale especialmente la política formativa; la cobertura de la formación es notablemente superior en las empresas más innovadoras. Se podría incluso afirmar que la formación cumple una función estratégica dentro de estas organizaciones al potenciar, a través de la cualificación del capital humano, la propia actividad innovadora. La relevancia de la actividad formativa se pone asimismo de manifiesto en la política que regula las promociones dentro de la empresa, donde el criterio fundamental por el que se establecen los ascensos es la formación interna.

El éxito de la innovación también se ha ligado al fomento de unos valores dentro de la organización orientados hacia la participación, la autonomía y la capacidad de tomar decisiones y aportar ideas por parte de los trabajadores. Sobre este punto, los resultados obtenidos sí confirman que son las empresas más innovadoras las que otorgan mayor importancia a este tipo de valores. La promoción de procesos creativos para resolver problemas, la evaluación de las ideas de los trabajadores y la participación y discusión abierta son valores considerados más importantes cuanto mayor es el nivel de innovación. Junto a ellos, también destaca la promoción de la innovación y el cambio. Sin embargo, aunque la autonomía de los trabajadores es valorada positivamente, no adquiere tanta relevancia como los valores anteriores.

Se puede concluir afirmando que en las organizaciones innovadoras los directivos sí otorgan una mayor importancia a aquellos valores que persiguen una mayor implicación de los trabajadores en la consecución de los objetivos empresariales, e incluso son favorables a un aumento de la autonomía en los puestos de trabajo. Sin embargo, en la práctica no suelen implementar las técnicas o sistemas adecuados para que tales valores se materialicen. Parece, pues, que el objetivo prioritario para estas organizaciones es aprovechar los conocimientos de los trabajadores para así mejorar los procesos de fabricación y los resultados de la empresa, sin que ello presuponga necesariamente una mayor autonomía en el puesto de trabajo y una delegación del poder para tomar decisiones organizativas, o para ejercer un mayor control sobre la producción. Las prerrogativas propias de la dirección se siguen manteniendo. 


\section{BIBLIOGRAFÍA}

BARBA ARAGÓN, M. I., ARAGÓN SÁNCHEZ, A. y SANZ VALLE, R. (2000): «Condicionantes de la formación en las PYMES industriales», Economía Industrial, no 334 , pp. 35-44.

BALDWIN, J. R. y JOHNSON, J. (1996): «Business strategies in more-and less-innovative firms in Canada», Research Policy, vol. 25, pp. 785-804.

BECKER, B. E. y HUSELID, M. A. (1998): «High performance work systems and firm performance: A synthesis of research and managerial implications», Research in Personnel and Human Resources Management, vol. 16, pp. 53-101.

BHOOVARAGHAVAN, S.; VASUDEVAN, A y RAJAN, C. (1996): «Resolving the process vs product innovation dilemma: a consumer choice theoretic approach», $M a$ nagement Science, vol. 42, no 2, pp. 232-246.

BRUNET, I. y ALARCÓN, A. (2007): «Calidad y recursos humanos del sector hotelero en Cataluña», Revista Internacional de Sociología, vol. LXV, no 47, pp. 73-98.

BUESA, M.; BAUMERT, T; HEIJS, J. y MARTÍNEZ, M. (2002): «Los factores determinantes de la innovación: un análisis econométrico sobre las regiones españolas», Economía Industrial, no 347 , pp. 67-84.

CALVO, J. L. (2000): «La distribución del gasto empresarial en innovación entre las comunidades autónomas españolas (1994-1998)», Economía Industrial, nº 334, pp. 7180.

CASTILLO, J. J.; JIMÉNEZ, V. y SANTOS, M. (1991): «Nuevas formas de organización del trabajo y de implicación directa en España», Revista Española de Investigaciones Sociológicas, no ${ }^{-56}$, pp. 115-141.

CASTILLO, J. J. (2000): «La emergencia de nuevos modelos productivos. Producción ligera e intensificación del trabajo en España», Trabajo y Sociedad, Vol. II, no2, www.geocities.com/trabajoysaciedad/S-THOYFINAL.htm.

CORONADO GUERRERO, D. y ACOSTA SERÓ, M. (1999): «Innovación tecnológica y desarrollo regional», Información Comercial Española, $\mathrm{n}^{\mathrm{o}}$ 781, pp. 103-116.

COSTA, M. T., N. DUCH y LLADÓS, J. (2001): «Determinantes de la innovación y efectos sobre la competitividad: el caso de las empresas textiles», Revista Asturiana de Economía, no 20, pp. 53-80.

DAMANPOUR, F. (1991): «Organizational innovation: A Meta-Analysis of effect o Determinants and Moderators», Academy of Management Journal, vol. 34, nº 3, pp. 555-590.

DAMANPOUR, F. y GOPALAKRISHNAN, S. (1998): «Theories of Organizational Structure and Innovation Adoption: the Role of Environmental Change», Journal of Engineering and Technology Management, vol. 15, nº 1, pp. 1-24.

DE LONG, D. W. y FAHEY, L. (2000): «Diagnosing cultural barriers to knowledge management», Academy of Management Executive, vol. 14, no 4, pp. 113-127.

DIÉGUEZ CASTRILLÓN, M. I. (2003): La formación empresarial ante la introducción de nuevas tecnologías de fabricación. El caso del sector metalmecánica gallego, Tesis Doctoral, Universidad de Vigo.

FARIÑAS, J. C., y HUERGO, E. (1999): «Tamaño empresarial, innovación y políticas de competencia», Economía Industrial, no 329 , pp. 67-80.

FERNÁNDEZ, E.; AVELLA, L. y FERNÁNDEZ, M. (2003): Estrategias de producción, Madrid, McGraw-Hill. 
GARCÍA ESPEJO, I. (2008): «Formación e innovación en las empresas industriales» $P A$ PERS, $\mathrm{n}^{\circ}$ 88, pp. 179-194.

GÓMEZ URANGA, M. J.; ZABALA ITURRIAGAGOITIA, M. y FERNÁNDEZ DE LUCIO, I. (2008): «Panorama de la innovación en España a través de la evolución de indicadores regionales», Economía Industrial, no 368, pp125-139.

GONZÁLEZ, A.; JIMÉNEZ, J. J. y SÁEZ, F. J. (1997): «Comportamiento innovador de las pequeñas y medianas empresas», Investigaciones Europeas de Dirección y Economía de la Empresa, Vol. 3, no 1, pp. 93-112.

GUARNIZO GARCÍA, J. V. y GUADAMILLAS GÓMEZ, F. (1998): «Innovación y desarrollo tecnológico en las empresas industriales españolas. Factores explicativos según la encuesta de estrategias empresariales», Economía Industrial, nº 319, pp. 4962.

GUMBAU ALBERT, M. (1997): «Análisis macroeconómico de los determinantes de la innovación: aplicación a las empresas industriales españolas», Revista Española de Economía, vol. 14, no 1, pp. 41-66.

HAILEY, V. H. (2001): «Breaking the mould? Innovation as a strategy for corporate renewal», International Journal Resource Management, vol. 12, n 7, pp. 1126-1140.

HAMMER, M. y CHAMPY, J. (1993): Reengineering the Corporation: A Manifesto for Business Revolution, Nueva York, Harper Business.

HARLEY, B. (1999): «The myth of empowerment: Work organization, hierarchy and employee autonomy in contemporary Australian workplaces», Work, Employment and Society, vol. 13, no 1 , pp. 41-66.

HUERGO, E. (2002): «Determinantes de la innovación tecnológica en la industria manufacturera española», Revista del Instituto de Estudios Económicos, nº 3, pp. 121142.

HUERTA ARRIBAS, E. (ed.) (2002): Los desafíos de la competitividad. La innovación organizativa y tecnológica en la empresa española, Bilbao, Fundación BBVA.

HUSELID, M. A. y BECKER, B. E. (1996): «Methodological Issues in Cross-Sectional and Panel Estimates of the Human Resources-Firm Perfomance Link», Industrial Relations, vol. 35, no 3, pp. 400-422.

INE (2007): Encuesta sobre Innovación Tecnológica en las Empresas.

LAHERA SÁNCHEZ, A. (2004): «La participación de los trabajadores en la calidad total: nuevos dispositivos disciplinarios de organización del trabajo», Revista Española de Investigaciones Sociológicas, $\mathrm{n}^{\mathrm{o}}$ 106, pp. 63-101.

LESTER, D. H. (1998): «Critical success factors for new product development», Research Technology Management, vol. 41, no 1, pp. 36-43.

MARÍN GARCÍA, J. A.; PARDO DEL VAL, M. y BONAVÍA MARTÍN, T. (2008): «La mejora continua como innovación incremental. El caso de una empresa industrial española», Economía Industrial, no 368 , pp. 155-167.

MARTÍNEZ LUCIO, M. y SIMPSON, D. (1993): «La dimensión social de las nuevas prácticas de gestión y su relevancia para la crisis de las relaciones laborales», Sociología del Trabajo, $\mathrm{n}^{-}$18, pp. 47-71.

MOLERO, J. y BUESA, M. (1995): «Configuración productiva y capacidad de innovación en la industria española», Información Comercial Española, no 743, pp. 59-83.

MORCILLO, P. (1997): Dirección estratégica de la tecnología e innovación. Un enfoque de competencias, Madrid, Civitas.

OSTERMAN, P. (1994): «How common is workplace transformation and who adopts it?, Industrial and Labor Relations Review», vol. 47, nº 2, pp. 173-188. 
— (2000): «Work reorganization in an era restructuring: trends in difusión and effects on employee welfare», Industrial and Labor Relations Review, Vol. 53, no 2, pp. 179196.

PANIAGUA LÓPEZ, J. A. (1999): «La calidad total: nuevas formas de nombrar el taylorismo», Sociología del Trabajo, no 37, pp. 41-58.

PCTI Asturias 2006-2009. www.pctiasturias.com

QUINN, R. E. y ROHRBAUCH, J. (1983): «A Spatial Model of Effectiveness Criteria: Toward a Competing Values Approach to Organizational Analysis», Management Science, vol. 29, no 3, pp. 363-377.

RUÍZ MERCADER, J. et al. (2004): «Innovación y cultura empresarial» www.acede.org/index_archivos/CDMurcia.

TOMÁS CARPI, J. A. y TORREJÓN VELARDIEZ, M. (2000-2001): «Flexibilidad productiva y organización del trabajo», Sociología del Trabajo, no 41, pp.101-125.

TUSHMAN, M. L. y O'REILLY III, Ch. A. (2002): Winning through innovation. A practical guide to leading organizational change and renewal, Boston, Harvard Business School Press.

URBANO PULIDO, D. y TOLEDANO GARRIDO, N. (2008): «Los proyectos innovadores en las PYMES españolas. Un estudio de casos múltiple», Economía Industrial, no 368 , pp. 213-225.

URRACA RUIZ, A. (1998): «I+D y recursos alternativos a la innovación en la industria española», Economía Industrial, no 319 , pp. 91-104.

VAN DER PANNE, G.; VAN BEERS, C. y KLEINKNECHT, A. (2003): «Success and failure of innovation: a literature review», International Journal of Innovation Management, Vol. 7, no 3, pp. 309-338.

VÁZQUEZ, J. A. y LOMBA, R. (2000): «La industria asturiana, un sector en transformación», Economía Industrial, no ${ }^{\circ} 335 / 336$, pp. 111-122.

VELASCO BALMASEDA, E. y ZAMANILLO ELGUEZABAL, I. (2008): «Evolución de las propuestas sobre el proceso de innovación: ¿qué se puede concluir de su estudio?, Investigaciones Europeas de Dirección y Economía de la Empresa, vol. 14, no 2, pp. 127-138.

VILLAVICENCIO, D. (2000): «La innovación en las empresas como espacio de análisis sociológico», Sociología del Trabajo, no 40, pp. 59-78.

YAGÜE, M. J. (1992): «La actividad innovadora de la PYME industrial española», Economía Industrial, no 284, pp. 153-159. 


\section{ANEXO}

Tabla 7. Importancia de los valores empresariales. Estadística descriptiva

\begin{tabular}{|c|c|c|c|c|c|}
\hline & & Media & $\begin{array}{l}\text { Desviación } \\
\text { Típica }\end{array}$ & Mínimo & Máximo \\
\hline Eficiencia & $\begin{array}{l}\text { - Nivel bajo } \\
\text { - Nivel Medio } \\
\text { - Nivel alto }\end{array}$ & $\begin{array}{l}4,21 \\
4,44 \\
4,54\end{array}$ & $\begin{array}{l}0,800 \\
0,649 \\
0,536\end{array}$ & $\begin{array}{l}2 \\
3 \\
3\end{array}$ & $\begin{array}{l}5 \\
5 \\
5\end{array}$ \\
\hline Productividad & $\begin{array}{l}\text { - Nivel bajo } \\
\text { - Nivel Medio } \\
\text { - Nivel alto }\end{array}$ & $\begin{array}{l}4,40 \\
4,46 \\
4,58\end{array}$ & $\begin{array}{l}0,660 \\
0,530 \\
0,563\end{array}$ & $\begin{array}{l}3 \\
3 \\
3\end{array}$ & $\begin{array}{l}5 \\
5 \\
5\end{array}$ \\
\hline Rentablilidad & $\begin{array}{l}\text { - Nivel bajo } \\
\text { - Nivel Medio } \\
\text { - Nivel alto }\end{array}$ & $\begin{array}{l}4,26 \\
4,41 \\
4,39\end{array}$ & $\begin{array}{l}0,836 \\
0,623 \\
0,644\end{array}$ & $\begin{array}{l}2 \\
3 \\
3\end{array}$ & $\begin{array}{l}5 \\
5 \\
5\end{array}$ \\
\hline Trabajo en equipo & $\begin{array}{l}\text { - Nivel bajo } \\
\text { - Nivel Medio } \\
\text { - Nivel alto }\end{array}$ & $\begin{array}{l}3,81 \\
4,17 \\
4,02\end{array}$ & $\begin{array}{l}0,900 \\
0,793 \\
0,777\end{array}$ & $\begin{array}{l}1 \\
2 \\
2\end{array}$ & $\begin{array}{l}5 \\
5 \\
5\end{array}$ \\
\hline Cohesión & $\begin{array}{l}\text { - Nivel bajo } \\
\text { - Nivel Medio } \\
\text { - Nivel altol }\end{array}$ & $\begin{array}{l}3,13 \\
3,51 \\
3,60\end{array}$ & $\begin{array}{l}1,020 \\
0,969 \\
1,091\end{array}$ & $\begin{array}{l}1 \\
1 \\
1\end{array}$ & $\begin{array}{l}5 \\
5 \\
5\end{array}$ \\
\hline Relaciones humanas & $\begin{array}{l}\text { - Nivel bajo } \\
\text { - Nivel Medio } \\
\text { - Nivel alto }\end{array}$ & $\begin{array}{l}3,73 \\
3,92 \\
4,03\end{array}$ & $\begin{array}{l}0,866 \\
0,732 \\
0,787\end{array}$ & $\begin{array}{l}1 \\
2 \\
2\end{array}$ & $\begin{array}{l}5 \\
5 \\
5\end{array}$ \\
\hline $\begin{array}{l}\text { Crecimiento, } \\
\text { expansión }\end{array}$ & $\begin{array}{l}\text { - Nivel bajo } \\
\text { - Nivel Medio } \\
\text { - Nivel alto }\end{array}$ & $\begin{array}{l}3,32 \\
3,71 \\
4,16\end{array}$ & $\begin{array}{l}0,936 \\
0,666 \\
0,790\end{array}$ & $\begin{array}{l}1 \\
2 \\
2\end{array}$ & $\begin{array}{l}5 \\
5 \\
5\end{array}$ \\
\hline $\begin{array}{l}\text { Excelencia, calidad } \\
\text { resultados }\end{array}$ & $\begin{array}{l}\text { - Nivel bajo } \\
\text { - Nivel Medio } \\
\text { - Nivel alto }\end{array}$ & $\begin{array}{l}4,04 \\
4,39 \\
4,44\end{array}$ & $\begin{array}{l}0,854 \\
0,621 \\
0,650\end{array}$ & $\begin{array}{l}2 \\
3 \\
3\end{array}$ & $\begin{array}{l}5 \\
5 \\
5\end{array}$ \\
\hline Innovación y cambio & $\begin{array}{l}\text { - Nivel bajo } \\
\text { - Nivel Medio } \\
\text { - Nivel alto }\end{array}$ & $\begin{array}{l}3,11 \\
3,66 \\
3,90\end{array}$ & $\begin{array}{l}1,013 \\
0,861 \\
0,912\end{array}$ & $\begin{array}{l}1 \\
2 \\
2\end{array}$ & $\begin{array}{l}5 \\
5 \\
5\end{array}$ \\
\hline Control & $\begin{array}{l}\text { - Nivel bajo } \\
\text { - Nivel Medio } \\
\text { - Nivel alto }\end{array}$ & $\begin{array}{l}3,77 \\
3,91 \\
3,97\end{array}$ & $\begin{array}{l}0,954 \\
0,737 \\
0,870\end{array}$ & $\begin{array}{l}1 \\
3 \\
1\end{array}$ & $\begin{array}{l}5 \\
5 \\
5\end{array}$ \\
\hline $\begin{array}{l}\text { Flexibilidad para } \\
\text { adaptarse }\end{array}$ & $\begin{array}{l}\text { - Nivel bajo } \\
\text { - Nivel Medio } \\
\text { - Nivel alto }\end{array}$ & $\begin{array}{l}3,85 \\
4,24 \\
4,31\end{array}$ & $\begin{array}{l}0,928 \\
0,746 \\
0,725\end{array}$ & $\begin{array}{l}1 \\
2 \\
3\end{array}$ & $\begin{array}{l}5 \\
5 \\
5\end{array}$ \\
\hline $\begin{array}{l}\text { Autonomía } \\
\text { trabajadores }\end{array}$ & $\begin{array}{l}\text { - Nivel bajo } \\
\text { - Nivel Medio } \\
\text { - Nivel alto }\end{array}$ & $\begin{array}{l}3,09 \\
3,41 \\
3,47\end{array}$ & $\begin{array}{l}1,061 \\
0,767 \\
0,971\end{array}$ & $\begin{array}{l}1 \\
1 \\
2\end{array}$ & $\begin{array}{l}5 \\
5 \\
5\end{array}$ \\
\hline $\begin{array}{l}\text { Participación, } \\
\text { discusión abierta }\end{array}$ & $\begin{array}{l}\text { - Nivel bajo } \\
\text { - Nivel Medio } \\
\text { - Nivel alto }\end{array}$ & $\begin{array}{l}2,94 \\
3,39 \\
3,47\end{array}$ & $\begin{array}{l}1,008 \\
0,836 \\
0,916\end{array}$ & $\begin{array}{l}1 \\
2 \\
2\end{array}$ & $\begin{array}{l}5 \\
5 \\
5\end{array}$ \\
\hline
\end{tabular}

EMPIRIA. Revista de Metodología de Ciencias Sociales. N. ${ }^{\circ}$ 17, enero-junio, 2009, pp. 63-90. ISSN: 1139-5737 


\begin{tabular}{|l|l|c|c|c|c|}
\hline & & Media & $\begin{array}{c}\text { Desviación } \\
\text { Típica }\end{array}$ & Mínimo & Máximo \\
\hline & - Nivel bajo & 3,30 & 1,137 & 1 & 5 \\
Evaluar ideas & - Nivel Medio & 3,62 & 0,763 & 2 & 5 \\
trabajadores & - Nivel alto & 3,73 & 0,848 & 1 & 5 \\
\hline & - Nivel bajo & 3,23 & 1,103 & 1 & 5 \\
Procesos creativos & - Nivel Medio & 3,55 & 0,891 & 2 & 5 \\
res. problemas & - Nivel alto & 3,69 & 0,883 & 1 & 5 \\
\hline
\end{tabular}

Fuente: Encuesta a empresas industriales 2005.

Tabla 8. PRUEBAS POST HOC. HSD de Tukey

\begin{tabular}{|c|c|c|c|c|}
\hline & $\begin{array}{l}\text { (I) Nivel de } \\
\text { innovación }\end{array}$ & $\begin{array}{l}\text { (J) Nivel de } \\
\text { innovación }\end{array}$ & $\begin{array}{c}\text { Diferencias } \\
\text { de medias (I-J) }\end{array}$ & Sig. \\
\hline \multirow{3}{*}{ Eficiencia } & - Nivel bajo & $\begin{array}{l}\text { Medio } \\
\text { alto } \\
\end{array}$ & $\begin{array}{l}-0,225 \\
-0,331 * \\
\end{array}$ & $\begin{array}{l}0,154 \\
0,026 \\
\end{array}$ \\
\hline & - Nivel Medio & $\begin{array}{l}\text { Bajo } \\
\text { Alto }\end{array}$ & $\begin{array}{r}0,225 \\
-0,106 \\
\end{array}$ & $\begin{array}{l}0,154 \\
0,638 \\
\end{array}$ \\
\hline & - Nivel alto & $\begin{array}{l}\text { Bajo } \\
\text { Medio }\end{array}$ & $\begin{array}{l}0,331^{*} \\
0,106\end{array}$ & $\begin{array}{l}0,026 \\
0,638\end{array}$ \\
\hline \multirow{3}{*}{ Cohesión } & - Nivel bajo & $\begin{array}{l}\text { Medio } \\
\text { alto }\end{array}$ & $\begin{array}{l}-0,375 \\
-0,471^{*}\end{array}$ & $\begin{array}{l}0,111 \\
0,043\end{array}$ \\
\hline & - Nivel Medio & $\begin{array}{l}\text { Bajo } \\
\text { Alto }\end{array}$ & $\begin{array}{l}0,375 \\
0,096\end{array}$ & $\begin{array}{l}0,111 \\
0,856\end{array}$ \\
\hline & - Nivel alto & $\begin{array}{l}\text { Bajo } \\
\text { Medio }\end{array}$ & $\begin{array}{l}0,471^{*} \\
0,096\end{array}$ & $\begin{array}{l}0,043 \\
0,856\end{array}$ \\
\hline \multirow{3}{*}{ Crecimiento, expansión } & - Nivel bajo & $\begin{array}{l}\text { Medio } \\
\text { alto }\end{array}$ & $\begin{array}{l}-0,389 * \\
-0,834 *\end{array}$ & $\begin{array}{l}0,021 \\
0,000\end{array}$ \\
\hline & - Nivel Medio & $\begin{array}{l}\text { Bajo } \\
\text { Alto }\end{array}$ & $\begin{array}{r}0,389^{*} \\
-0,445^{*}\end{array}$ & $\begin{array}{l}0,021 \\
0,005\end{array}$ \\
\hline & - Nivel alto & $\begin{array}{l}\text { Bajo } \\
\text { Medio }\end{array}$ & $\begin{array}{l}0,834^{*} \\
0,445^{*}\end{array}$ & $\begin{array}{l}0,000 \\
0,005\end{array}$ \\
\hline \multirow{3}{*}{$\begin{array}{l}\text { Excelencia, calidad } \\
\text { resultados }\end{array}$} & - Nivel bajo & $\begin{array}{l}\text { Medio } \\
\text { Alto }\end{array}$ & $\begin{array}{l}-0,357^{*} \\
-0,403^{*}\end{array}$ & $\begin{array}{l}0,016 \\
0,008\end{array}$ \\
\hline & - Nivel Medio & $\begin{array}{l}\text { Bajo } \\
\text { Alto }\end{array}$ & $\begin{array}{l}0,357 * \\
0,046\end{array}$ & $\begin{array}{l}0,016 \\
0,926\end{array}$ \\
\hline & - Nivel alto & $\begin{array}{l}\text { Bajo } \\
\text { Medio }\end{array}$ & $\begin{array}{l}0,403^{*} \\
0,046\end{array}$ & $\begin{array}{l}0,008 \\
0,926\end{array}$ \\
\hline
\end{tabular}

EMPIRIA. Revista de Metodología de Ciencias Sociales. N. ${ }^{\circ}$ 17, enero-junio, 2009, pp. 63-90. ISSN: 1139-5737 


\begin{tabular}{|c|c|c|c|c|}
\hline & $\begin{array}{l}\text { (I) Nivel de } \\
\text { innovación }\end{array}$ & $\begin{array}{l}\text { (J) Nivel de } \\
\text { innovación }\end{array}$ & $\begin{array}{c}\text { Diferencias } \\
\text { de medias (I-J) }\end{array}$ & Sig. \\
\hline \multirow{3}{*}{ Innovación y cambio } & - Nivel bajo & $\begin{array}{l}\text { Medio } \\
\text { alto }\end{array}$ & $\begin{array}{l}-0,549^{*} \\
-0,783^{*}\end{array}$ & $\begin{array}{l}0,004 \\
0,000\end{array}$ \\
\hline & - Nivel Medio & $\begin{array}{l}\text { Bajo } \\
\text { Alto }\end{array}$ & $\begin{array}{l}0,549^{*} \\
-0,235\end{array}$ & $\begin{array}{l}0,004 \\
0,325\end{array}$ \\
\hline & - Nivel alto & $\begin{array}{l}\text { Bajo } \\
\text { Medio }\end{array}$ & $\begin{array}{l}0,783^{*} \\
0,235\end{array}$ & $\begin{array}{l}0,000 \\
0,325\end{array}$ \\
\hline \multirow{3}{*}{$\begin{array}{l}\text { Flexibilidad para } \\
\text { adaptarse }\end{array}$} & - Nivel bajo & $\begin{array}{l}\text { Medio } \\
\text { alto }\end{array}$ & $\begin{array}{l}-0,390^{*} \\
-0,066\end{array}$ & $\begin{array}{l}0,021 \\
0,008\end{array}$ \\
\hline & - Nivel Medio & $\begin{array}{l}\text { Bajo } \\
\text { Alto }\end{array}$ & $\begin{array}{l}0,456^{*} \\
0,066\end{array}$ & $\begin{array}{l}0,021 \\
0,886\end{array}$ \\
\hline & - Nivel alto & $\begin{array}{l}\text { Bajo } \\
\text { Medio }\end{array}$ & $\begin{array}{l}-0,314 \\
-0,380\end{array}$ & $\begin{array}{l}0,008 \\
0,886\end{array}$ \\
\hline \multirow{3}{*}{$\begin{array}{l}\text { Participación, discusión } \\
\text { abierta }\end{array}$} & - Nivel bajo & $\begin{array}{l}\text { Medio } \\
\text { alto }\end{array}$ & $\begin{array}{l}-0,451^{*} \\
-0,531^{*}\end{array}$ & $\begin{array}{l}0,020 \\
0,007\end{array}$ \\
\hline & - Nivel Medio & $\begin{array}{l}\text { Bajo } \\
\text { Alto }\end{array}$ & $\begin{array}{l}0,451^{*} \\
-0,080\end{array}$ & $\begin{array}{l}0,020 \\
0,872\end{array}$ \\
\hline & - Nivel alto & $\begin{array}{l}\text { Bajo } \\
\text { Medio }\end{array}$ & $\begin{array}{l}0,531 * \\
0,080\end{array}$ & $\begin{array}{l}0,007 \\
0,872\end{array}$ \\
\hline \multirow{4}{*}{$\begin{array}{l}\text { Evaluar ideas } \\
\text { trabajadores }\end{array}$} & - Nivel bajo & $\begin{array}{l}\text { Medio } \\
\text { alto }\end{array}$ & $\begin{array}{l}-0,318 \\
-0,427 *\end{array}$ & $\begin{array}{l}0,136 \\
0,038\end{array}$ \\
\hline & - Nivel Medio & $\begin{array}{l}\text { Bajo } \\
\text { Alto }\end{array}$ & $\begin{array}{r}0,318 \\
-0,109 \\
\end{array}$ & $\begin{array}{l}0,136 \\
0,776\end{array}$ \\
\hline & - Nivel alto & $\begin{array}{l}\text { Bajo } \\
\text { Medio }\end{array}$ & $\begin{array}{l}0,427 * \\
0,109\end{array}$ & $\begin{array}{l}0,038 \\
0,776\end{array}$ \\
\hline & - Nivel bajo & $\begin{array}{l}\text { Medio } \\
\text { alto }\end{array}$ & $\begin{array}{l}-0,323 \\
-0,463^{*}\end{array}$ & $\begin{array}{l}0,153 \\
0,031\end{array}$ \\
\hline \multirow[t]{2}{*}{$\begin{array}{l}\text { Procesos creativos para } \\
\text { resolver problemas }\end{array}$} & - Nivel Medio & $\begin{array}{l}\text { Bajo } \\
\text { Alto }\end{array}$ & $\begin{array}{r}0,323 \\
-0,140 \\
\end{array}$ & $\begin{array}{l}0,153 \\
0,685\end{array}$ \\
\hline & - Nivel alto & $\begin{array}{l}\text { Bajo } \\
\text { Medio }\end{array}$ & $\begin{array}{l}0,463^{*} \\
0,140\end{array}$ & $\begin{array}{l}0,031 \\
0,685\end{array}$ \\
\hline
\end{tabular}

* Diferencias estadísticamente significativas

Fuente: Encuesta a empresas industriales 2005.

\section{RESUMEN}

En este artículo se analiza si la innovación dentro de las empresas va acompañada de la implantación de nuevas formas de organización del trabajo y de políticas de personal que impliquen una gestión más participativa de los recursos humanos. Asimismo, se abordan las relaciones entre la capacidad innovadora de las empresas y el fomento por parte de la dirección de valores dirigidos hacia una ma- 
yor participación, compromiso y autonomía de los trabajadores. El análisis empírico se basa en la información obtenida por medio de una encuesta realizada a industrias de más de 30 trabajadores. Los resultados obtenidos a partir de la aplicación de diferentes técnicas de análisis indican que en las organizaciones innovadoras sí se otorga mayor importancia a los valores que fomentan la participación, la iniciativa y la autonomía de los trabajadores. Sin embargo, los resultados también señalan que en tales empresas se ha avanzado poco en la implementación de los cambios organizativos y mecanismos necesarios para que dichos valores se materialicen en la práctica.

\title{
PALABRAS CLAVE
}

Empresas innovadoras, organización de la producción, valores empresariales.

\begin{abstract}
In this article, we analyze whether innovation in companies is related to the introduction of new forms of labour organization and personnel policies that imply more participative human resource management. At the same time, we will tackle the relations between firms' innovative capacities and the managements' promotion of values oriented towards higher participation, commitment and autonomy of workers. The empirical analysis is based on a survey carried out in companies of more than 30 workers. The results obtained after applying different techniques of analysis indicate that the innovative organizations do attribute greater importance to values that promote workers' participation, initiative and autonomy. However, the same results also demonstrate that these companies have progressed little in the implementation of organizational modifications and mechanisms needed to be able to put these values into practice.
\end{abstract}

\section{KEYWORDS}

Innovative firms, organization of production, business values 